\title{
Tipificación fágica de aislados de Salmonella enteritidis de muestras clínicas, alimentarias y avícolas en Chile ${ }^{1}$
}

\author{
Soledad Prat, ${ }^{2}$ Alda Fernández, ${ }^{2}$ Alberto Fica, ${ }^{3}$ Jorge Fernández, ${ }^{2}$ \\ Marcela Alexandre ${ }^{4}$ e Ingrid Heitmann ${ }^{2}$
}

RESUMEN Desde 1994 Chile ha sido afectado por una extensa epidemia de infecciones por Salmonella enteritidis (S. enteritidis). Para conocer la diversidad de fuentes infectantes, el posible origen de la epidemia y las relaciones epidemiológicas entre aislados clínicos, alimentarios y avícolas, se realizó la tipificación fágica de 310 muestras de S. enteritidis de origen clínico recolectadas entre los años 1975 y 1996 junto con 47 aislados alimentarios obtenidos durante brotes de S. enteritidis y 27 cepas aisladas en estudios de vigilancia de establecimientos de avicultura. En total, en las muestras clínicas se identificaron 13 fagotipos, dos aislados no pudieron tipificarse y uno se consideró atípico. Los tipos fágicos que se identificaron con mayor frecuencia fueron el $1(56,8 \%)$ y el $4(31,3 \%)$, seguidos de los tipos $8(4,8 \%)$ y $28(1,9 \%)$. La presencia regional y temporal de estos fagotipos indica que ha habido cambios importantes en su distribución: en los primeros años de recolección solo se registraron los fagotipos 8 y 28 , que desaparecieron hacia 1980 para reaparecer de forma aislada en 1996. A partir de la expansión paulatina de S. enteritidis que se inició en 1988, se empezaron a aislar en las zonas central y sur del país fagos del tipo 4, que no se habian encontrado anteriormente en Chile. En 1991 emergió como predominante en la zona norte el fagotipo 1, tampoco registrado previamente. En los aislados alimentarios se identificaron solamente los tipos 1 y 4, que fueron también los más comunes en los aislados avícolas. La tipificación fágica de S. enteritidis es de utilidad para orientar el análisis epidemiológico de las infecciones por este agente patológico.

Palabras clave Salmonella enteritidis, fagotipificación, epidemia, alimentos.

Las infecciones humanas por Salmonella enteritidis (Salmonella enterica sub-

1 Este trabajo fue financiado por el proyecto 1980912 del Fondo Nacional de Desarrollo Científico y Tecnológico (Fondecyt).

2 Instituto de Salud Pública, Subdepartamento de Microbiología y Unidad de Desarrollo, Santiago, Chile.

3 Toda la correspondencia debe enviarse a este autor, a la siguiente dirección: Programa de Microbiología, Facultad de Medicina, Universidad de Chile. Av. Independencia 1027, Santiago, Chile. Correo electrónico: afica@machi.med.uchile.cl

4 Servicio de Salud Metropolitano del Ambiente, Santiago, Chile. especie enterica serotipo enteritidis [S. enteritidis]), que se observaban inicialmente solo en países desarrollados, han ido extendiéndose progresivamente a otras regiones (1). Estas infecciones se transmiten principalmente por el consumo de huevos o productos avícolas contaminados e inadecuadamente preparados (2-4). En varios países como Argentina, Brasil, y Chile, este agente patógeno empezó a conocerse a mediados de los años 80 como resultado de inesperados brotes epidémicos. Hoy en día, en esos países se re- gistran regularmente tasas de S. enteritidis típicas de la infección endémica $(1,5)$.

En 1994 comenzó en Chile una epidemia de infecciones por $S$. enteritidis que aumentó en 3 000\% las bajas tasas observadas en épocas anteriores (6). Los primeros casos se registraron en el norte del país y 3 a 4 años más tarde ya se habían esparcido por la mayor parte del territorio chileno. Hasta ahora no se conocen razones que puedan explicar claramente la emergencia de las infecciones por S. enteritidis en Chile, si 
bien podría aducirse la introducción de ese agente por aves destinadas a la reproducción importadas de países afectados. Otra posible explicación es que los establecimientos avícolas se hayan contaminado con cepas naturales del país.

Los estudios epidemiológicos sobre S. enteritidis requieren métodos de tipificación que permitan discriminar y separar cepas no relacionadas entre sí. Con este propósito se han utilizado diferentes métodos moleculares, pero los resultados han sido variables. La electroforesis de campos pulsados (7-11), la reacción en cadena de la polimerasa con cebadores arbitrarios (12) y la ribotipificación $(7-9,13)$ han sido algunas de las estrategias moleculares utilizadas que han revelado una gran variedad en su capacidad discriminatoria. Esta variabilidad obliga a que la selección de una estrategia sobre otra se base en el trabajo empírico y al mismo tiempo limita los estudios comparativos entre aislados identificados en diferentes países. Actualmente, el método de tipificación bacteriana de la $S$. enteritidis más utilizado corresponde a la tipificación fágica, lo que se explica por su estandarización, la facilidad de montaje y porque la mayor parte de los aislados son tipificables y, además, los resultados son fácilmente reproducibles. Su limitación más importante radica en su baja capacidad discriminatoria debido a la predominancia de unos pocos fagotipos en una población bacteriana.

La tipificación fágica de aislados nacionales de $S$. enteritidis es de interés debido a que esta técnica de marcación ha estado restringida a unos pocos países, en su mayor parte desarrollados. Ello ha impedido conocer cuáles poblaciones bacterianas están involucradas en las nuevas zonas afectadas. Más aún, esta técnica de marcación podría indicar si solo unos pocos fagotipos están asociados con la actual epidemia o si, como se sospecha, varios tipos podrían estar circulando a partir de diferentes fuentes infectantes. Además, la comparación de aislados clínicos, alimentarios y avícolas podría mostrar la relación epidemiológica de estos. Por último, la comparación de los fagotipos que actualmente circulan con los que se han observado en épocas anteriores podría indicar el posible origen local de la epidemia o su importación del exterior.

\section{MATERIALES Y MÉTODOS}

Para este trabajo se utilizó parte de una colección de aislados del género Salmonella iniciada en el Instituto de Salud Pública (ISP) de Chile en 1975. La muestra incluyó 310 aislados clínicos de $S$. enteritidis identificados en deposiciones o muestras de sangre y confirmados como tales por el Centro de Referencia de Enterobacterias del ISP, de acuerdo con criterios establecidos (14). Esos aislados se recolectaron entre 1975 y 1996 y representan 21\% del total de 1445 aislados de $S$. enteritidis acumulados hasta 1996. La muestra incluye $94 \%$ de los aislados preepidémicos, es decir, casi todos los obtenidos entre 1975 y 1993.

La mayor parte de las muestras clínicas (281 ó 90,6\%) se obtuvieron de coprocultivos. Quince muestras provinieron de hemocultivos $(4,8 \%), 4$ de orina $(1,3 \%)$ y 10 de otro origen o de origen desconocido. También se incluyeron en el análisis 47 aislados de $S$. enteritidis de muestras alimentarias y 27 aislados identificados en 1995 o 1996 en establecimientos avícolas y enviados al ISP por el Servicio Agrícola y Ganadero.

La tipificación fágica se llevó a cabo desde marzo a diciembre de 1999, de acuerdo con el procedimiento descrito por la doctora L. R. Ward del Centro Colaborador de la OMS de Referencia e Investigación de Salmonella en Londres. Esta metodología se ha estandarizado y su descripción se ha publicado previamente (15).

Todos los aislados clínicos se analizaron por el método de dilución en placa para estudiar su susceptibilidad a los antimicrobianos cloranfenicol, ampicilina, cefotaxima, ciprofloxacino y trimetoprima-sulfametoxazol. Se utilizaron los criterios de corte y procedimientos publicados por el National Committee for
Clinical Laboratory Standards de los Estados Unidos de América (16).

Las comparaciones estadísticas que se señalan en el texto se calcularon con ayuda del programa Statistica (versión 4.5, Statsoft, Inc.).

\section{RESULTADOS}

\section{Aislados clínicos}

En las 310 muestras de origen clínico se identificaron 13 fagotipos. Dos cepas no fueron tipificables y una mostró un resultado atípico, lo que representa en conjunto $1 \%$ de los aislados clínicos. Los fagotipos más frecuentes fueron el 1 y el 4, que representaron $56,8 \%$ y $31,3 \%$ de los aislados, respectivamente, seguidos de los fagotipos 8,28 y 7. Se observaron con menor frecuencia otros tipos, ninguno de los cuales sobrepasó 0,7\% del total. Los fagotipos 1 y 4 se aislaron de $88,1 \%$ de las muestras clínicas. Otros fagotipos tales como los 2, 3, 5, 20, 21, 7a, 13a y $4 \mathrm{a}$, aparecieron esporádica e infrecuentemente en uno o dos aislados cada uno (cuadro 1).

Hubo cambios importantes en la distribución de fagotipos durante el

\section{CUADRO 1. Distribución de tipos fágicos en 310 aislados clínicos de Salmonella en- teritidis, Chile, 1975-1996}

\begin{tabular}{lrr}
\hline Fagotipo & Número & Porcentaje \\
\hline 1 & 176 & 56,8 \\
4 & 97 & 31,3 \\
8 & 15 & 4,8 \\
28 & 6 & 1,9 \\
7 & 3 & 1,0 \\
5 & 2 & 0,7 \\
$13 a$ & 2 & 0,7 \\
2 & 1 & 0,3 \\
3 & 1 & 0,3 \\
21 & 1 & 0,3 \\
$7 a$ & 1 & 0,3 \\
$4 a$ & 1 & 0,3 \\
$20 a$ & 1 & 0,3 \\
Atípico & 1 & 0,3 \\
No tipificable & 2 & 0,7 \\
Total & 310 & 100 \\
\hline
\end{tabular}


período analizado (figura 1). Los aislados clínicos de $S$. enteritidis fueron muy infrecuentes al inicio de la colección del ISP y hasta fines de los años ochenta. En ese período se identificaron solo dos fagos de los tipos 8 y 28 . Posteriormente, en las muestras recibidas por el ISP no se volvió a aislar $S$. enteritidis hasta 1988, cuando se aislaron fagotipos no observados previamente. El retorno de S. enteritidis se limitó inicialmente a aislados del fagotipo 4 y tres años más tarde aparecieron también cepas del fagotipo 1. Se observaron infrecuentemente otros fagos, pero no los tipos iniciales, salvo en casos esporádicos de enfermedad asociados con el fagotipo 8 en 1996. Desde 1994, la epidemia de S. enteritidis ha estado asociada exclusivamente con aislados pertenecientes a los fagotipos 4 y 1 .

El análisis regional indica la predominancia de aislados del fagotipo 1 de S. enteritidis en el norte de Chile y del fagotipo 4 en el centro y el sur del país (figura 2). Casi 94\% de los aislados del fagotipo 1 se originaron en el norte, mientras que $92 \%$ de los aislados del fagotipo 4 provienen del centro y del sur (cuadro 2).

Por el método de dilución en placa no se detectaron cepas resistentes a cloranfenicol, cefotaxima, ciprofloxacino ni trimetoprima-sulfametoxazol (datos no mostrados). Entre las muestras clínicas analizadas, se identificaron solo dos cepas resistentes a la ampicilina $(0,6 \%$ del total). Esos aislados de los fagotipos 4 y 1 se identificaron en 1991 y 1995, respectivamente, y provenían de dos servicios de salud diferentes del norte del país.

\section{Muestras alimentarias}

Las muestras alimentarias incluidas en este estudio se han identificado progresivamente desde 1993 en conexión con brotes de toxicoinfección. Los fagotipos 4 y 1 fueron los únicos aislados de 47 muestras: 20 y 27, respectivamente. La mayor parte de los alimentos involucrados en esos brotes (38,3\%) eran productos hechos a base de huevos
FIGURA 1. Distribución de fagotipos en aislados clínicos de Salmonella enteritidis según el aislamiento. Chile, 1975-1996

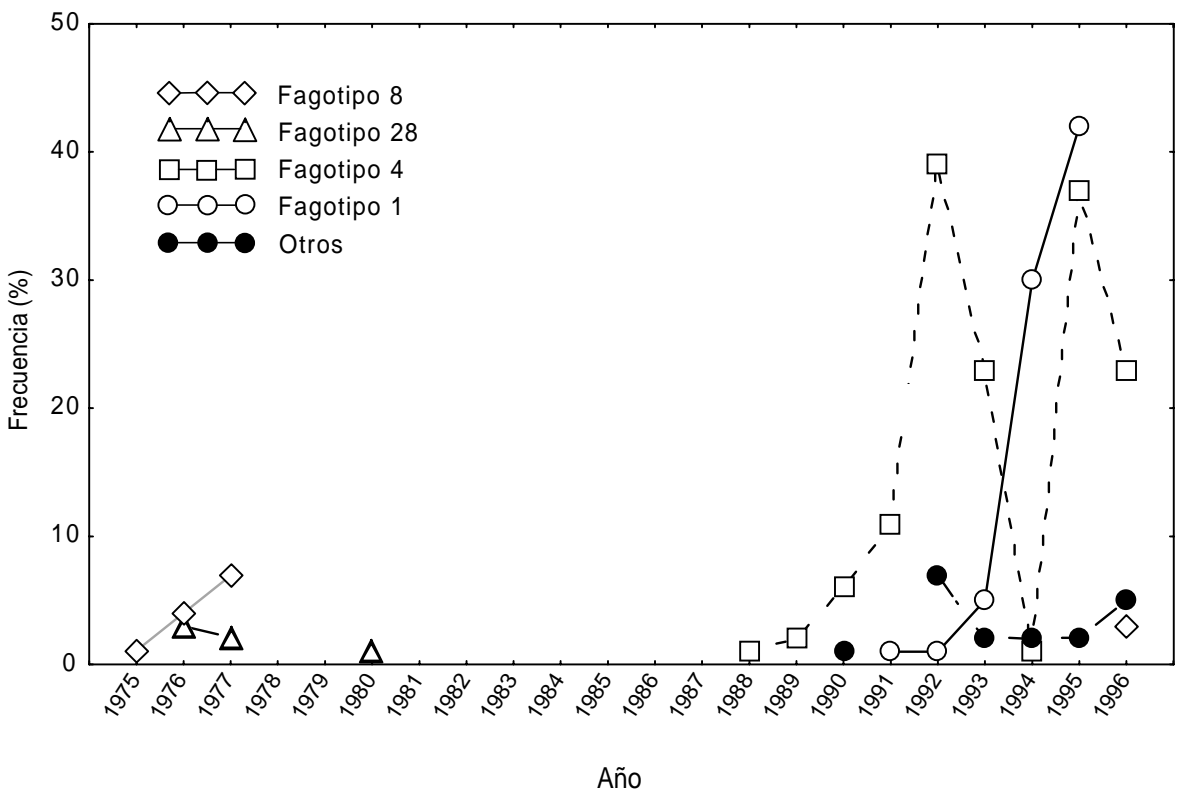

como la mayonesa o las tortas de merengue. En el contexto de los brotes aparecen con mucho menor frecuencia los alimentos que contienen carne de

ave o entrañas de pollo (6,4\%). La distribución proporcional de los fagotipos 4 y 1 fue similar en ambos tipos de alimentos (cuadro 3).

FIGURA 2. Distribución regional de aislados clínicos de Salmonella enteritidis pertenecientes a los fagotipos 4 y 1. Chile, 1975-1996

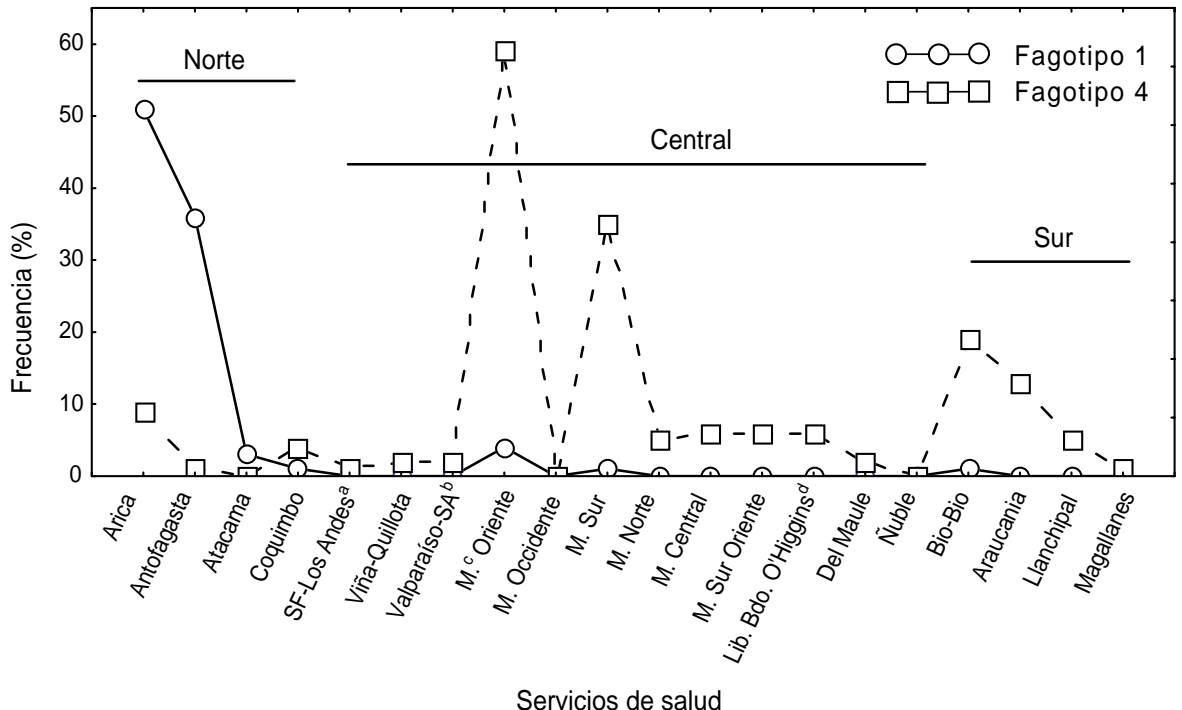

a SF-Los Andes = Servicio de Salud San Felipe, Los Andes.

b Valparaíso-SA = Servicio de Salud Valparaíso, San Antonio.

${ }^{c} \mathrm{M}=$ Servicio metropolitano respectivo.

d Lib. Bdo. O'Higgins = Servicio de Salud Libertador Bernardo O'Higgins. 
CUADRO 2. Distribución porcentual de aislados clínicos de Salmonella enteritidis pertenecientes a los fagotipos 1 ó 4, por región de Chile, 1975-1996

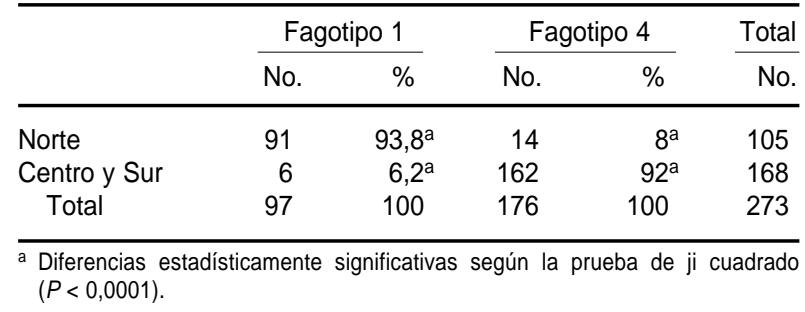

\section{Aislados avícolas}

Durante 1995 y 1996, el Servicio Agrícola y Ganadero remitió al ISP aislados de S. enteritidis obtenidos en estudios de vigilancia de establecimientos avícolas. Lamentablemente, no se contó con información sobre el origen mayor parte de los casos, la información sobre el origen anatómico de los aislados estaba incompleta. En 27 aislados examinados, se reconocieron cuatro fagotipos, entre los que predominaron 14 del tipo $1(51,9 \%)$ y 10 del tipo $4(37 \%)$. Algunos de estos aislados provenían de muestras de las cloacas o los ovarios de gallinas de postura. Se identificaron también cepas pertenecientes a los fagotipos 2 y 7 en uno y dos casos, respectivamente.

\section{DISCUSIÓN}

Los resultados presentados en este trabajo indican que la tipificación fágeográfico de las muestras $y$, en la gica de aislados de $S$. enteritidis es útil para orientar el trabajo epidemiológico, a pesar de su bajo poder discriminatorio. La epidemia de infecciones por $S$. enteritidis vigente en Chile se debe a dos fagotipos diferentes que tienen una clara distribución regional dentro del país. En el norte del país predomina el fagotipo 1 y en las regiones centro y sur, el fagotipo 4 . Los aislamientos del fagotipo 4 precedieron a los del tipo 1 en tres años, aunque actualmente ambos tipos coexisten. La epidemia que se inició en 1994 no estuvo asociada con la reemergencia de antiguos fagotipos de S. enteritidis; los tipos 1 y 4 no se habían detectado previamente en una colección que abarca varios años de registro.

Hasta ahora, la tipificación fágica de aislados de $S$. enteritidis se ha concentrado principalmente en los países desarrollados de Europa, Asia, América y Australia (17-22). Hay pocos informes provenientes de países en desarrollo (23) y, a pesar del aumento notable de estas infecciones en varios
CUADRO 3. Distribución de fagotipos en aislados alimentarios de Salmonella enteritidis, Chile, 1975-1996

\begin{tabular}{|c|c|c|c|c|c|c|}
\hline \multirow[b]{2}{*}{ Alimento } & \multicolumn{2}{|c|}{ Fagotipo 1} & \multicolumn{2}{|c|}{ Fagotipo 4} & \multicolumn{2}{|c|}{ Total } \\
\hline & No. & $\%$ & No. & $\%$ & No. & $\%$ \\
\hline \multicolumn{7}{|l|}{ Que contiene huevo: } \\
\hline tortas, mayonesa, etc. & 9 & 19,1 & 9 & 19,1 & 18 & 38,2 \\
\hline $\begin{array}{l}\text { Derivado de carne de } \\
\text { ave o entrañas de pollo }\end{array}$ & 1 & 21 & 2 & 43 & 3 & 6.4 \\
\hline Ensaladas & 7 & 14,9 & 4 & 8,5 & 11 & 23,4 \\
\hline Otros tipos de carne & 3 & 6,4 & 2 & 4,3 & 5 & 10,7 \\
\hline Otros alimentos & 7 & 14,9 & 3 & 6,4 & 10 & 21,3 \\
\hline Total & 27 & 57,4 & 20 & 42,6 & 47 & 100 \\
\hline
\end{tabular}

países latinoamericanos, en estos solo se han publicado algunos trabajos relacionados con Brasil (5). La distribución de los fagotipos tiene una clara variación geográfica con predominio de los tipos 4, 1, 6 y 8 en Europa (17, $19,21)$ y de los tipos 4,8 y 13 a en Canadá $(17,22)$. En los Estados Unidos de América se presenta un perfil parecido, con un predominio de los fagotipos 8, 13a, 13 y 14b y una baja frecuencia de los fagotipos 4 y 1 (18). Estos datos indican que los aislados pertenecientes al fagotipo 1 son más propios del continente europeo, mientras que el fagotipo 4 puede considerarse de distribución universal. En Brasil también, los aislados del fagotipo 4 han reemplazado los antiguos aislados del fagotipo 8 (5). La situación en Chile refleja el predominio universal de los aislados del tipo 4 y el perfil europeo del fagotipo 1, no habitual en las colecciones americanas. La concordancia en la distribución de los fagotipos predominantes en Chile con los fagotipos observados en otras latitudes y la ausencia de los fagotipos tradicionales 8 y 28 en la actual epidemia sugieren una contaminación de los establecimientos avícolas nacionales por $S$. enteritidis de fuentes externas. Ello podría haber ocurrido por la entrada al país de aves destinadas a la reproducción importadas de Europa, Estados Unidos o Brasil. A pesar del probable origen externo de los contaminantes iniciales, actualmente la epidemia tiene un claro origen doméstico, tal como queda demostrado por el continuo aislamiento de este serotipo en los establecimientos nacionales de avicultura y por la concordancia entre los fagotipos observados en el medio clínico y los que se han identificado en esos establecimientos.

En la actual epidemia, la predominancia de fagotipos no implica necesariamente que solo dos líneas clonales estén causando la mayor parte de las infecciones. El gran número de fagotipos que se identifican en aislamientos clínicos sugiere diversas fuentes infectantes. Además, los estudios preliminares desarrollados en nuestro laboratorio con electroforesis de campos 
pulsados indican que los aislados de los dos fagotipos más frecuentes presentan una gran diversidad filogenética y no constituyen grupos homogéneos. ${ }^{5}$ Esta misma diversidad indica que en la comunidad circulan varias fuentes infectantes de $S$. enteritidis, lo que hace imposible erradicarlas en la práctica. Por ello los esfuerzos de control deberán basarse en regular las etapas productivas y de distribución, complementados con campañas de educación dirigidas a los consumidores.

La concordancia entre los fagotipos de interés en el medio clínico y los que se han identificado en alimentos liga-

\footnotetext{
5 Ebensperger G, Fica A, Fernández J, Prat S, Fernández $A$, Alexandre $M$, et al. Evaluación del poder discriminatorio de la fagotipificación y de diferentes técnicas moleculares en aislamientos de Salmonella enteritidis: estudio preliminar. Trabajo presentado en el XVI Congreso Chileno de Infectología, Pucón, Chile, 1999.
}

dos a brotes y en muestras avícolas destaca la importancia de los productos avícolas en la epidemiología y la transmisión de las infecciones por $S$. enteritidis. En estudios paralelos se han identificado también aislados de S. enteritidis en muestras de carne de ave y huevos tomadas al azar en Santiago (24). Cabe recalcar la gran importancia del hallazgo de $S$. enteritidis en la cloaca y los ovarios de aves de postura. La presencia de S. enteritidis en la cloaca facilita la contaminación del huevo durante la postura, cuando la cáscara es aún permeable, y la presencia de este agente en muestras de ovario subraya la relevancia que tiene la inoculación vertical (transovárica) del huevo en la epidemiología de estas infecciones (25). Esta forma de contaminación impide controlar las infecciones por $S$. enteritidis mediante el simple lavado o la desinfección de la superficie de los huevos.

\section{REFERENCIAS}

1. Rodrigue DC, Tauxe RV, Rowe B. International increase in Salmonella enteritidis: a new pandemic? Epidemiol Infect 1990;105(1):21-27.

2. Mishu B, Griffin PM, Tauxe R, Cameron D, Huthcheson RH, Schaffner W. Salmonella enteritidis gastroenteritis transmitted by intact chicken eggs. Ann Intern Med 1991;115(3): 190-194.

3. Hennessy TW, Hedberg CW, Slustker L, White KE, Besser-Wiek JM, Moen ME, et al. A national outbreak of Salmonella enteritidis infections from ice cream. The Investigation Team. N Engl J Med 1996;334(20):1281-1286.

4. Henzler DJ, Ebel E, Sanders J, Kradel D, Mason J. Salmonella enteritidis in eggs from commercial chicken layer flocks implicated in human outbreaks. Avian Dis 1994;38(1):37-43.

5. Irino K, Fernandes SA, Tavechio AT, Neves BC, Dias AM. Progression of Salmonella enteritidis phage type 4 strains in São Paulo State, Brazil. Rev Inst Med Trop São Paulo. 1996; 38(3):193-196.

6. Fica A, Fernández A, Prat $S$, Figueroa $\mathrm{O}$, Gamboa R, Tsunekawa I, et al. Salmonella enteritidis, un patógeno emergente en Chile. Rev Med Chile 1997;125(5):544-551.

7. Thong K-L, Ngeow Y-F, Altwegg M, Navaratnam P, Pang T. Molecular analysis of Salmonella enteritidis by pulsed-field gel electrophoresis and ribotyping. J Clin Microbiol 1995; 33(5):1070-1074.
8. Liebisch B, Schwarz S. Molecular typing of Salmonella enterica subsp. enterica serovar enteritidis isolates. J Med Microbiol 1996;44(1):52-59.

9. Olsen JE, Skov MN, Threlfall EJ, Brown DJ. Clonal lines of Salmonella enterica serotype enteritidis documented by IS200-, ribo-, pulsedfield gel electrophoresis and RFLP typing. J Med Microbiol 1994;40(1):15-22.

10. Suzuki $Y$, Ishihara M, Matsumoto M, Arakawa S, Saito M, Ishikawa N, et al. Molecular epidemiology of Salmonella enteritidis. An outbreak and sporadic cases studied by means of pulsed-field gel electrophoresis. J Infection 1995;31(3):211-217.

11. Powell NG, Threlfall EJ, Chart H, Rowe B. pulsed-field gel electrophoresis: potential for epidemiological surveillance. FEMS Microbiol Lett 1994;119(1-2):193-198.

12. Fadl AA, Nguyen AV, Khan MI. Analysis of Salmonella enteritidis isolates by arbitrarily primed PCR. J Clin Microbiol 1995;33(4): 987-989.

13. Martinetti G, Altwegg M. rRNA gene restriction patterns and plasmid analysis as a tool for typing Salmonella enteritidis. Res Microbiol 1990;141(9):1151-1162.

14. Popoff MY, Le Minor L. Antigenic formulas of the Salmonella serovars. WHO Collaborating Centre for Reference and Research on Salmonella, Institute Pasteur, Paris, 1997. Subdivision of Salmonella enteritidis PT4 by
La emergencia de las infecciones por S. enteritidis en Chile coincide con la reducción gradual de las infecciones asociadas con S. typhi y otras salmonelas entéricas. Las tasas nacionales de fiebre tifoidea han disminuido de valores que excedían de 120 por 100000 a comienzos de los años ochenta hasta los de 9 por 100000 en 1997 (26). Por contraste, las tasas de casos de diarrea por S. enteritidis han aumentado de menos de 0,35 casos por 100000 en el período de 1975-1992 a 3,41 por 100000 en 1994, un incremento de más de $3000 \%$ (6).

Agradecimientos. Los autores agradecen a la Organización Panamericana de la Salud y al Centro Colaborador de la OMS de Referencia e Investigación de Salmonella en Londres su apoyo logístico y la entrega de la colección de fagos necesaria para llevar a cabo este trabajo.
15. Ward LR, De Sa JDH, Rowe B. A phagetyping scheme for Salmonella enteritidis. Epidemiol Infect 1987;99:291-294.

16. National Committe for Clinical Laboratory Standards 1998;18(1); and 1997;17(1).

17. Ward LR. IFEPT Report, 1996. 8th International Congress of Bacteriology and Applied Microbiology. Jerusalem, August, 1996.

18. Hickman-Brenner FW, Stubbs AD, Farmer III JJ. Phage typing of Salmonella enteritidis in the United States. J Clin Microbiol 1991;29(12): 2817-2823.

19. Lukinmaa S, Schildt R, Rinttila $T$, Siitonen A. Salmonella enteritidis phage types 1 and 4 : pheno- and genotypic epidemiology of recent outbreaks in Finland. J Clin Microbiol 1999; 37(7):2176-2182.

20. Terajima J, Nakamura A, Watanabe H. Epidemiological analysis of Salmonella enterica enteritidis isolates in Japan by phage-typing and pulsed-field gel electrophoresis. Epidemiol Infect 1998;120(3):223-229.

21. Nastasi A, Mammina C. Epidemiology of Salmonella enterica serotype enteritidis infections in southern Italy during the years 1980-1994. Res Microbiol 1996;147(5):393-403.

22. Poppe C. Salmonella enteritidis in Canada. Int J Food Microbiol 1994;21(1-2):1-5.

23. Boonmar S, Bangtrakulnonth A, Pornrunangwong S, Terajima J, Watanabe H, Kaneko K, et al. Epidemiological analysis of Salmonella 
enteritidis isolates from humans and broiler chickens in Thailand by phage typing and pulsed-field gel electrophoresis. J Clin Microbiol 1998;36(4):971-974.

24. Alexandre M, Pozo C, González V, Martínez MC, Prat S, Fernández A, et al. Detección de Salmonella enteritidis en muestras de vigilancia de productos avícolas de consumo humano en la Región Metropolitana. Rev Med Chile 2000; $128: 1075-1083$.

25. Gast RK, Beard CW. Production of Salmonella enteritidis-contaminated eggs by experimentally infected hens. Avian Dis 1990; 34(2): $438-46$.
26. Zunnino E. Vacunación antitífica. Rev Chil Infect 1999;16(Supl. 1):82-84.

Manuscrito recibido el 7 de abril de 1999 y aceptado para publicación, tras revisión, el 2 de noviembre de 2000.

ABSTRACT Since 1994 an extensive epidemic of infections with Salmonella enteritidis (S. enteritidis) has affected Chile. In order to understand the diversity of infective sources, the possible origin of the epidemic, and the epidemiological relationships between clinical, food, and poultry isolates, we carried out phage typing of three groups of samples: 1) 310 S. enteritidis clinical samples collected between 1975 and 1996, 2) 47 food isolates obtained during S. enteritidis outbreaks, and 3) 27 strains isolated in surveillance studies of poultry-raising establishments. With the clinical samples, a total of 13 phage types were identified, 2 isolates could not be typed, and 1 was considered atypical. The phage types that were identified most frequently were $1(56.8 \%)$ and $4(31.3 \%)$, trailed by type $8(4.8 \%)$ and type $28(1.9 \%)$. Over time and in different regions of the country there were major changes in the distribution of the phage types. In the first years of collection the only phage types registered were 8 and 28 , which disappeared around 1980 and then began reappearing sporadically in 1996. With the gradual S. enteritidis expansion that started in 1988, in the central and southern areas of the country phage type 4 began to appear; that type had not been found before in Chile. In 1991 in the northern area of the country phage type 1 began to predominate; it was another type that had not been reported before in Chile. In the food isolates the only phage types identified were 1 and 4, which were also the most common in the poultry isolates. Phage typing of $S$. enteritidis has proved to be useful in guiding the epidemiological analysis of the infections caused by this pathogen.

In neither acute, infectious, nor chronic disease is a complete understanding of cause required for prevention. Smallpox was prevented before isolation of the bacterium; lung cancer can be prevented before identification of the specific carcinogen in cigarrete smoke. When an infectious disease is transmitted or maintained because of attitudes, behavior or surroundings, a purely germ-oriented approach is unlikely to provide effective control.

[En ningún tipo de enfermedad, sea aguda, infecciosa o crónica, se requiere una comprensión absoluta de la causa para prevenir la enfermedad. La viruela se previno antes de aislar el virus; el cáncer del pulmón se puede prevenir antes de identificar la sustancia cancerígena específica en el humo del cigarrillo. Cuando una enfermedad infecciosa se transmite o mantiene debido a actitudes, conductas 0 factores ambientales, es poco probable que un enfoque exclusivo en el microorganismo pueda rendir un control eficaz.]

Elizabeth Barrett-Connor "Infectious and chronic disease epidemiology: separate and unequal" American Journal of Epidemiology, 1979:109 